\title{
Editorial
}

\section{Nanomaterials for Energy and Environmental Applications}

\author{
Sesha Srinivasan, ${ }^{1}$ Arunachalanadar M. Kannan, ${ }^{2}$ Nikhil Kothurkar, ${ }^{3}$ \\ Yehia Khalil, ${ }^{4}$ and Sarada Kuravi ${ }^{5}$ \\ ${ }^{1}$ Florida Polytechnic University, 4700 Research Way, Lakeland, FL 33805, USA \\ ${ }^{2}$ The Polytechnic School, Ira A. Fulton Schools of Engineering, Arizona State University, Mesa, AZ 85212, USA \\ ${ }^{3}$ Department of Chemical Engineering and Materials Science, Amrita Vishwa Vidyapeetham, Amritanagar, Coimbatore 641112, India \\ ${ }^{4}$ United Technologies Research Corporation, 411 Silver Lane, East Hartford, CT 06118, USA \\ ${ }^{5}$ Department of Mechanical and Aerospace Engineering, New Mexico State University, Las Cruces, NM 88003, USA
}

Correspondence should be addressed to Sesha Srinivasan; ssrinivasan@flpoly.org

Received 19 November 2015; Accepted 23 November 2015

Copyright (C) 2015 Sesha Srinivasan et al. This is an open access article distributed under the Creative Commons Attribution License, which permits unrestricted use, distribution, and reproduction in any medium, provided the original work is properly cited.

Nanomaterials enabled technologies have been seamlessly integrated into applications such as aviation and space, chemical industry, optics, solar hydrogen, fuel cell, batteries, sensors, power generation, aeronautic industry, building/construction industry, automotive engineering, consumer electronics, thermoelectric devices, pharmaceuticals, and cosmetic industry. Clean energy and environmental applications often demand the development of novel nanomaterials that can provide shortest reaction pathways for the enhancement of reaction kinetics. Understanding the physicochemical, structural, microstructural, surface, and interface properties of nanomaterials is vital for achieving the required efficiency, cycle life, and sustainability in various technological applications. Nanomaterials with specific size and shape such as nanotubes, nanofibers/nanowires, nanocones, nanocomposites, nanorods, nanoislands, nanoparticles, nanospheres, and nanoshells to provide unique properties can be synthesized by tuning the process conditions.

The major objective of this special issue is to bring out the salient research paradigms of nanomaterials and their potential impacts on clean energy generation, storage, utilization, waste heat recovery, environmental detoxification, and disinfection and advocate the process sustainability. The research papers accepted after thorough review process for publication in this issue are briefly narrated below. The potential clean energy and environmental applications where the nanomaterials are employed and reported in this special issue are for (a) microcombustion and thermoelectric devices, (b) compressed natural gas reservoirs fabrication, (c) high temperature shale well drilling, and (d) water purification by removal of arsenic $(\mathrm{V})$ and bisphenol.

D. McNally et al. reported the design of a thermoelectric device using approximately $8 \mathrm{~nm}$ platinum nanoparticles as catalysts for microcombustion applications. The asdeveloped Pt nanoparticles seem to play a major role in controlling the fuel conversion (in case of ethanol, methane, propane, and butane) and the heat production rate as well. For the fabrication of natural gas reservoirs, it is reported by G. J. Pavani et al. that polymeric nanocomposites tandem with carbon fiber composites (i) improved the strengths of the liner, (ii) reduced the final weight of the reservoir, and (iii) decreased the gas permeability as well. These nanocomposites based compressed natural gas (CNG) reservoirs are potential alternative for vehicular applications. X. Yang et al. have successfully developed stabilization processes of silica nanoparticles based brine muds for the shale gas wells drilling applications. These nanoparticles brine muds (NPBMs) enhanced the mud systems' capabilities such as physical plugging of nanoparticles, balanced chemical activity with inorganic salts $(\mathrm{NaCl}, \mathrm{KCl}$, etc.), rational drilling mud density, and the wellbore stability. Other potential applications of water treatment residual nanoparticles (nWTR) as sorbents for the arsenic removal is demonstrated by E. Elkhatib et al. According to the authors, the nWTR consists 
of iron, silicon, calcium, and aluminum in either oxide or hydroxide forms. It is claimed that the nWTR showed at least 16 times higher reactivity in arsenic (V) removal when compared to their bulk counterparts. Similar to arsenic removal mentioned above, K. Mphahlele et al. have shown the removal of bisphenol A (BPA) from aqueous solution using Fe/N-CNTs- $\beta$-cyclodextrin nanocomposites. All the above research papers are original and are well aligned with the scope of this special issue.

\section{Acknowledgments}

We thank all the contributing authors and reviewers for their help in bringing out this special issue. We also thank the editorial board for the smooth process flow and publication.

Sesha Srinivasan Arunachalanadar M. Kannan Nikhil Kothurkar Yehia Khalil

Sarada Kuravi 

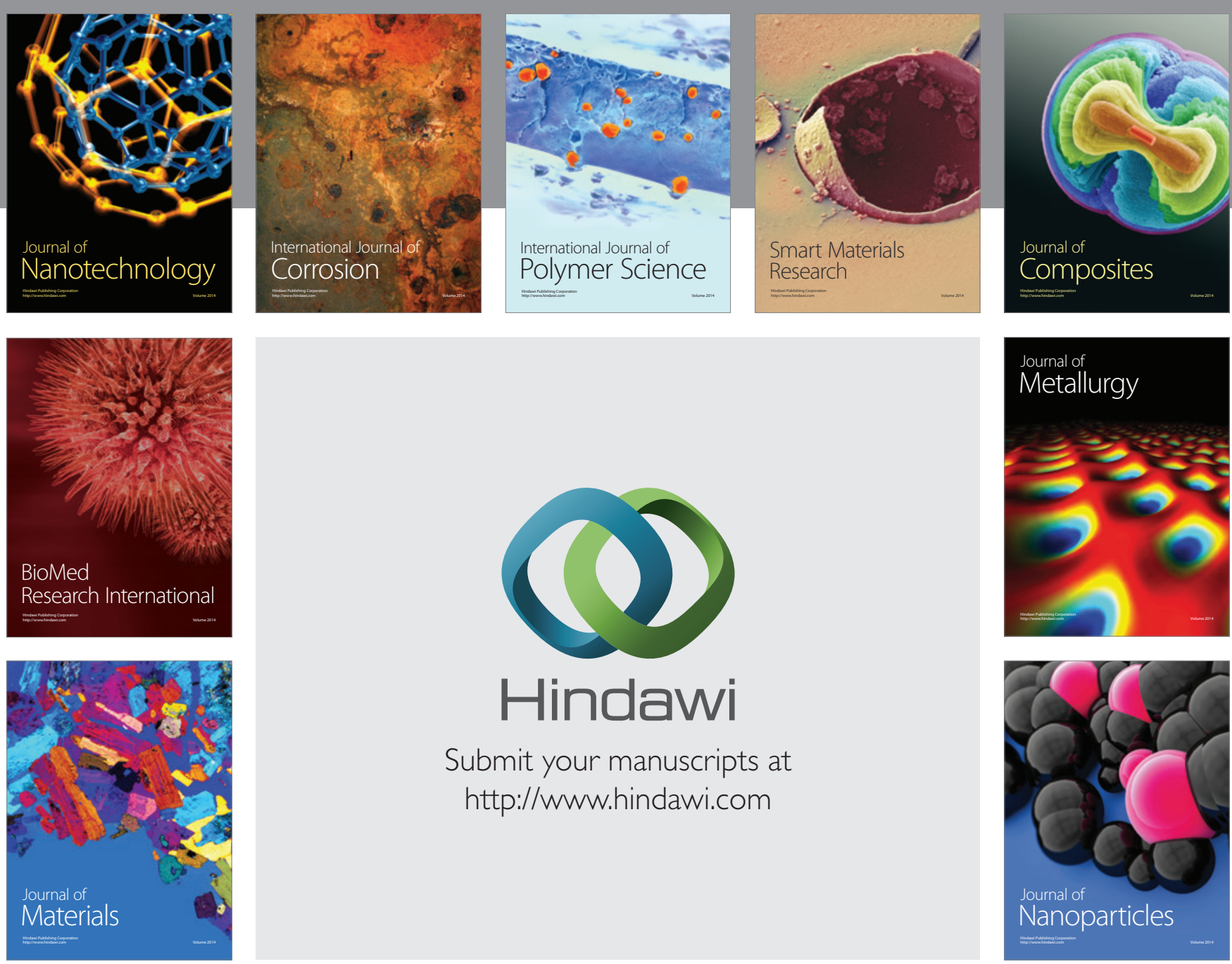

Submit your manuscripts at http://www.hindawi.com
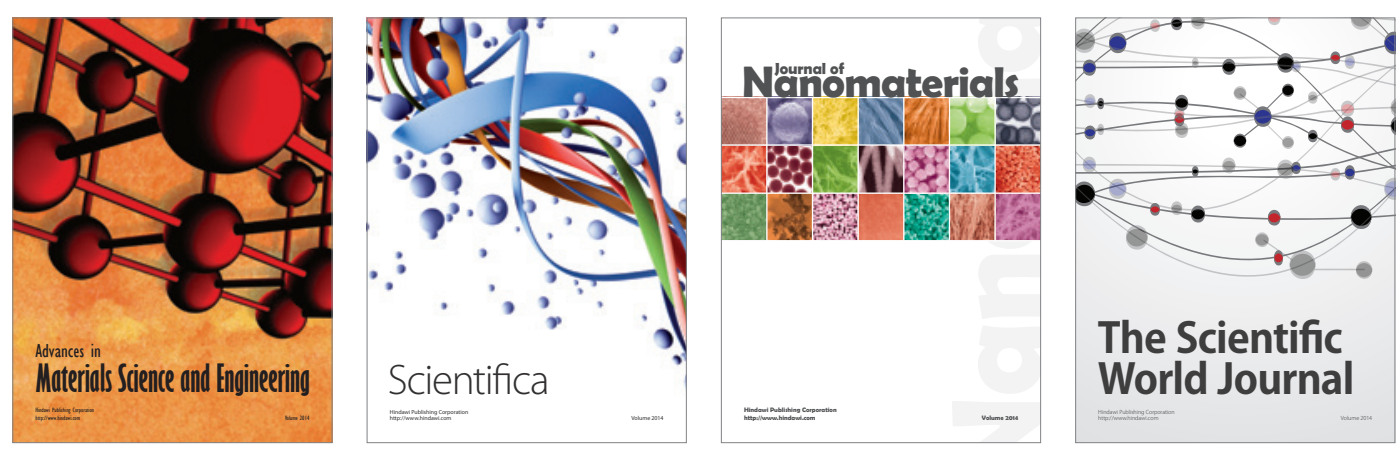

\section{The Scientific World Journal}
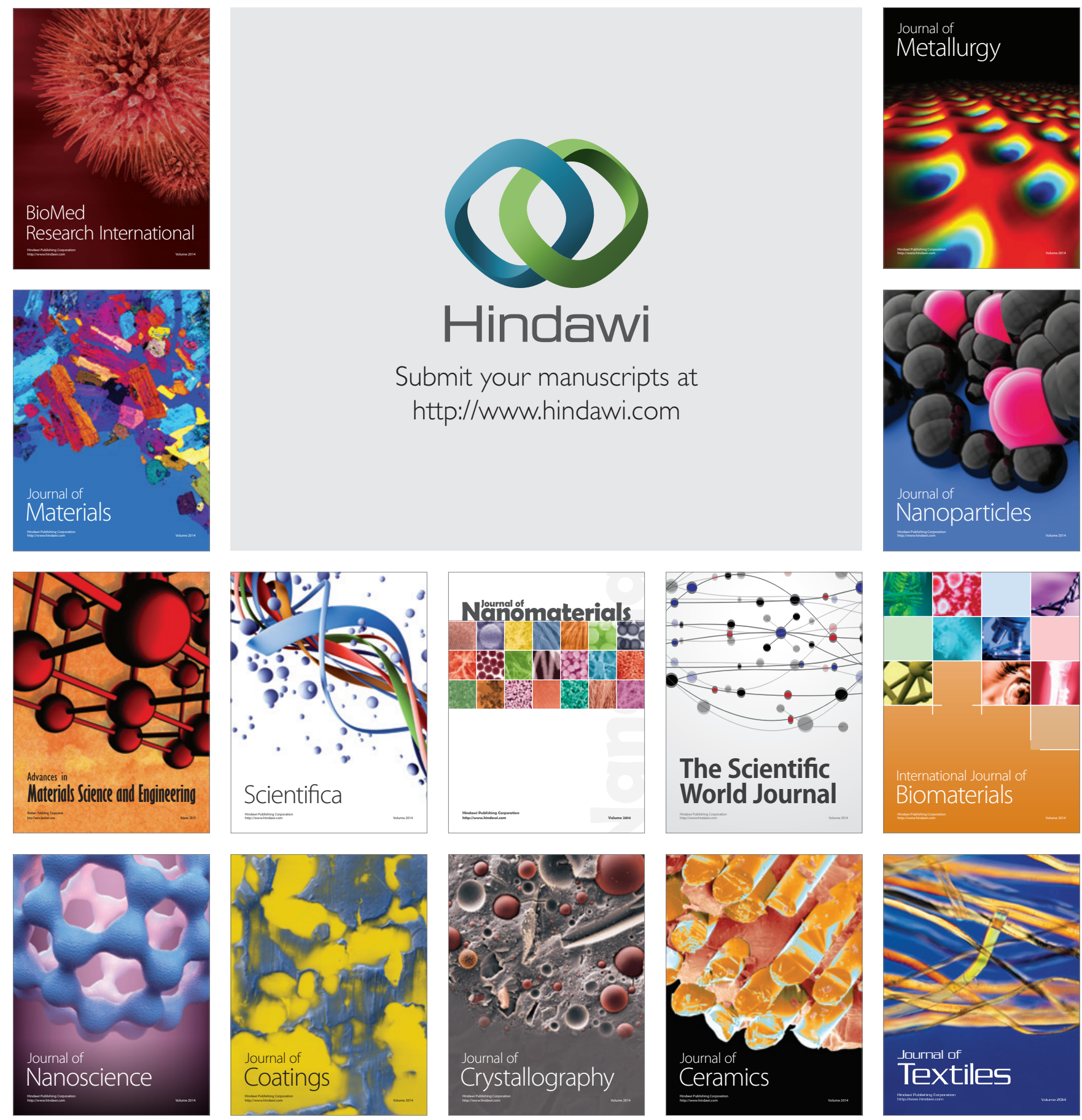